\title{
Thermal ions dilution and ITG suppression in ASDEX
}

\section{Upgrade ion ITBs}

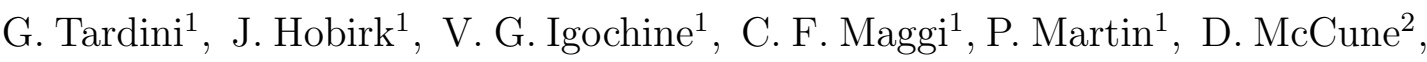
A. G. Peeters ${ }^{1}$, A. C. C. Sips ${ }^{1}$, A. Stäbler ${ }^{1}$, J. Stober ${ }^{1}$, and the ASDEX Upgrade Team $^{1}$

${ }^{1}$ Max-Planck-Institut für Plasmaphysik, Euratom Association, Boltzmannstrasse 2, D-85748 Garching, Germany

${ }^{2}$ Princeton Plasma Physics Laboratory, Princeton University, Princeton, NJ08543, USA

Abstract. Internal Transport Barriers (ITBs) in the ion channel in the tokamak ASDEX Upgrade allow for high energy confinement but collapse after only several energy confinement times. In this paper we show that in most cases the ITB phase is terminated clearly before the first ELM burst, thereby ruling out the ELMs as main trigger of the ITB collapse. For the first time, the ITB formation and sustainment are found to be associated with a mechanism of transport suppression based on thermal ions dilution by the injected fast ions. Interestingly, such ITBs do not require reversed magnetic shear. The linear growth rate of the Ion Temperature Gradient driven mode is computed as a function of the fast ion fraction with gyrokinetic stability analysis. Monte Carlo simulations predict the fast ion population to be above the gyrokinetic critical fraction in a region consistent with the experimental ITB width. The density threshold documented for the onset of ASDEX Upgrade ion ITBs is explained. The role of $T_{i} / T_{e}$ and of the plasma sheared rotation for ITB sustainment are analysed. The stabilisation mechanism presented here is consistent with the observed ITB lifetime of the order of the beam slowing down time. A possible runaway mechanism leading to ITB collapse is described. Finally, the relevance of this particular ITB scheme for ITER is discussed. 


\section{G. Tardini et al.}

\section{Introduction}

Anomalous transport due to microturbulence dominates the core energy confinement in tokamak devices, limiting the achievable density and temperature gradients. In particular, ion heat transport is commonly believed to be dominated by the Ion Temperature Gradient (ITG) driven mode, as demonstrated for ASDEX Upgrade H-mode discharges [1]. The mode can be suppressed locally to give rise to Internal Transport Barriers (ITBs), leading to improved energy confinement and high bootstrap current fractions. ITB feasibility and sustainment are, therefore, crucial issues for steady state tokamak operation in the next step device ITER [2]. For this reason ITBs have attracted considerable interest over the last 20 years, theoretically as well as experimentally. In particular, a variety of ITBs has been obtained in most present day tokamaks with different methods and properties [3]. In ASDEX Upgrade both electron [4] and ion ITBs [5] are obtained. In this paper we focus on ion ITBs produced by applying strong NBI power in discharges with low plasma density $\left(n_{e}\right)$. Until several years ago pre-heating was thought to be a necessary procedure to develop an ion ITB in ASDEX Upgrade, since the reversed q-profile was believed to be the key ingredient for ITG suppression. Those ITBs, however, were not reproducible [5], until it was found that low plasma density plays a major role in the ITB formation [6]. For this reason, presently ITBs are reliably produced by applying high NBI power at once, shortly before the current flat top [5]. The typical power is $10 \mathrm{MW}$ while $n_{e}$ ranges between 2 and $310^{19} \mathrm{~m}^{-3}$. High energy confinement is achieved, with central ion temperature $\left(T_{i}\right)$ up to $25 \mathrm{keV}$, associated with a moderate improvement in bootstrap current and significant $\beta_{N}$ values up to 2.9. The main limitation of this scenario is the short lifetime of the order of the beam slowing down time $\left(\tau_{s d}\right)[7]$, which is about $100 \mathrm{~ms}$ in the discharges considered here, comparable to the energy confinement time.

In this paper we identify the fast ion concentration to be the key ingredient of the ion ITB formation, providing a theoretical explanation of the experimental phenomenology. In the next section we discuss the robustness of the ITB scenario with respect to different plasma parameters such as the plasma current and the magnetic field. The NBI timing is also varied with respect to the beginning of the current flat top. In section 3. we investigate the timing of the ITB collapse with particular interest in a possible correlation with the onset of type I ELMs. The MHD activity, which is different from discharge to discharge, is also briefly analysed. In section 4 . the stabilisation mechanism of the ITG is introduced, with simplified 1D transport models highlighting the role of 


\section{Article: Thermal ions dilution and ITG suppression in ASDEX Upgrade ion ITBs}

the fast ion fraction. In section 5. we derive the critical fast ions fraction for the ITG suppression by means of linear gyrokinetic stability analysis. The result is compared to the time dependent prediction of the fast ion population according to Monte Carlo simulations, presented in section 6.. A runaway mechanism, possibly responsible for the ITB loss, is suggested. The role of the thermal ions dilution in several tokamaks and for different scenarios is described in section 7., including a discussion about the applicability of this ITB scheme to ITER. Finally, conclusions are drawn.

\section{ITB performance and timing}

The comprehensive diagnostics equipement of ASDEX Upgrade allows to resolve the ITB in time as well as in space. In particular the Charge eXchange Recombination Spectroscopy (CXRS) is used for $T_{i}$ and toroidal velocity $\left(v_{t o r}\right)$ measurements. Since 2004 a fast CXRS system is available for the core plasma, with a time resolution down to $10 \mathrm{~ms}$. To obtain a good signal to noise ratio, a time resolution of $20 \mathrm{~ms}$ has been set up. The fast CXRS system measures at 8 different radial locations at a time. A second CXRS system has 13 channels and a time resolution of $70 \mathrm{~ms} . T_{e}$ profiles are measured with the Electron Cyclotron Emission with a sampling rate of $32 \mathrm{kHz}$. Thomson scattering as well as interferometry measurements are available for the $n_{e}$ profiles. A set of experiments has been performed following the heating scheme described in [5] with one-step NBI input, in order to understand and possibly delay or at least mitigate the ITB collapse.

In all discharges, $n_{e}$ is kept as low as possible, limited only by the machine conditioning. The NBI power is 10 MW. The magnetic field, the plasma current, the current ramp-up rate and the timing of the NBI onset with respect to the beginning of the current flat top have been varied, in order to avoid or delay the MHD activity which might account for the termination of the good confinement phase. Throughout the scans ASDEX Upgrade ITBs prove to be quite insensitive to the different $q_{95}$ and to the different NBI timing. Transport barriers are reproducible and show the same time evolution, shown in Fig. 1. ITBs develope about 50-70 ms after the beams are switched on. They reach their maximum $\mathrm{R} / \mathrm{L}_{T_{i}}$ some $100 \mathrm{~ms}$ later, eventually decaying into an H-mode discharge after another 50-150 ms. Typical temperature profiles during and after the ITB phase are shown in Fig. 2, plasma density and toroidal velocity profiles in Fig. 3. While the ion temperature profile has clearly a region with strong $\nabla T_{i}$, no ITBs are observed in the electron temperature nor plasma density. The 


\section{G. Tardini et al.}

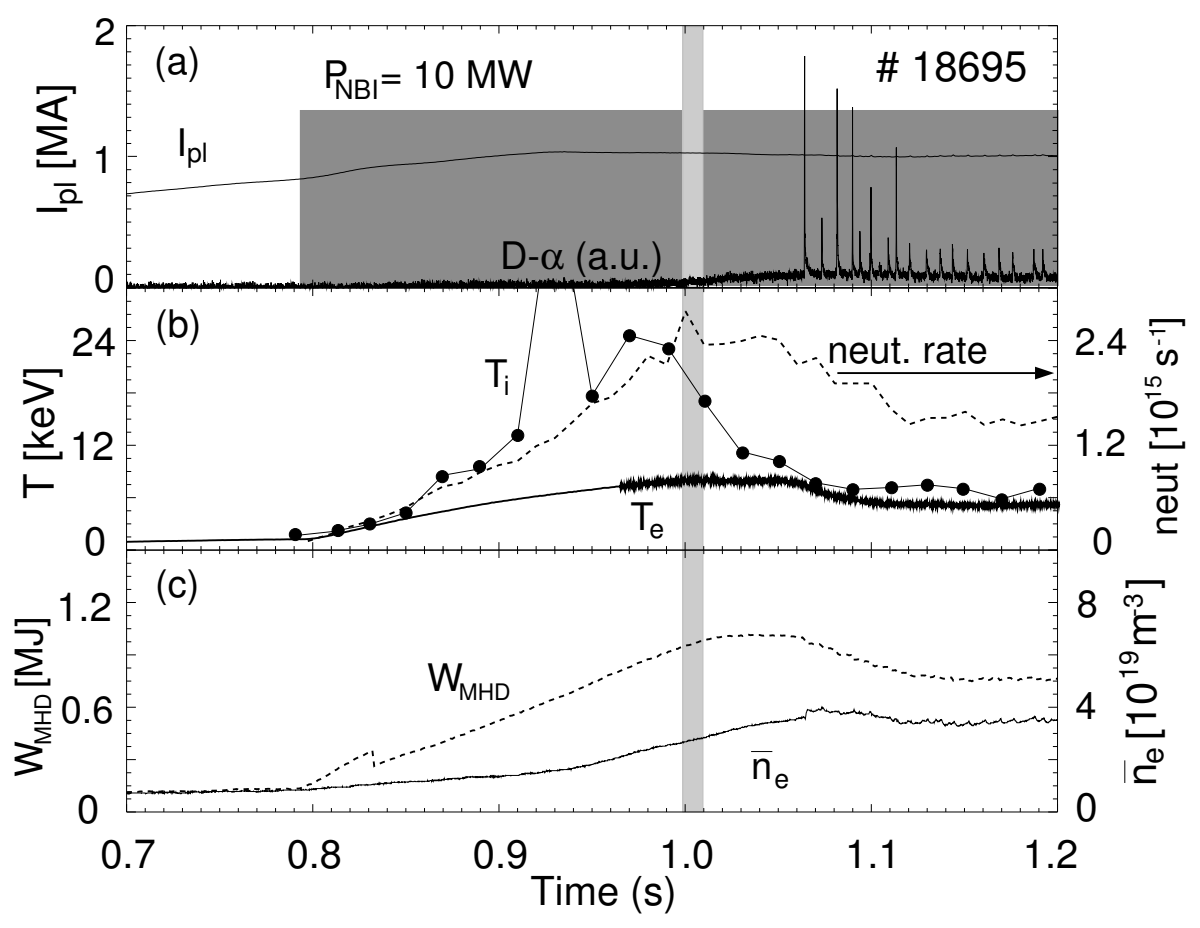

Figure 1. Time traces of a typical ITB discharge: (a) Plasma current, NBI power (shaded), divertor $\alpha$ signal. (b) $T_{i}$, $T_{e}$ and neutron rate (dashed line). (c) Stored energy (dashed line) and line averaged density. The shaded vertical region marks the beginning of the ITB loss.
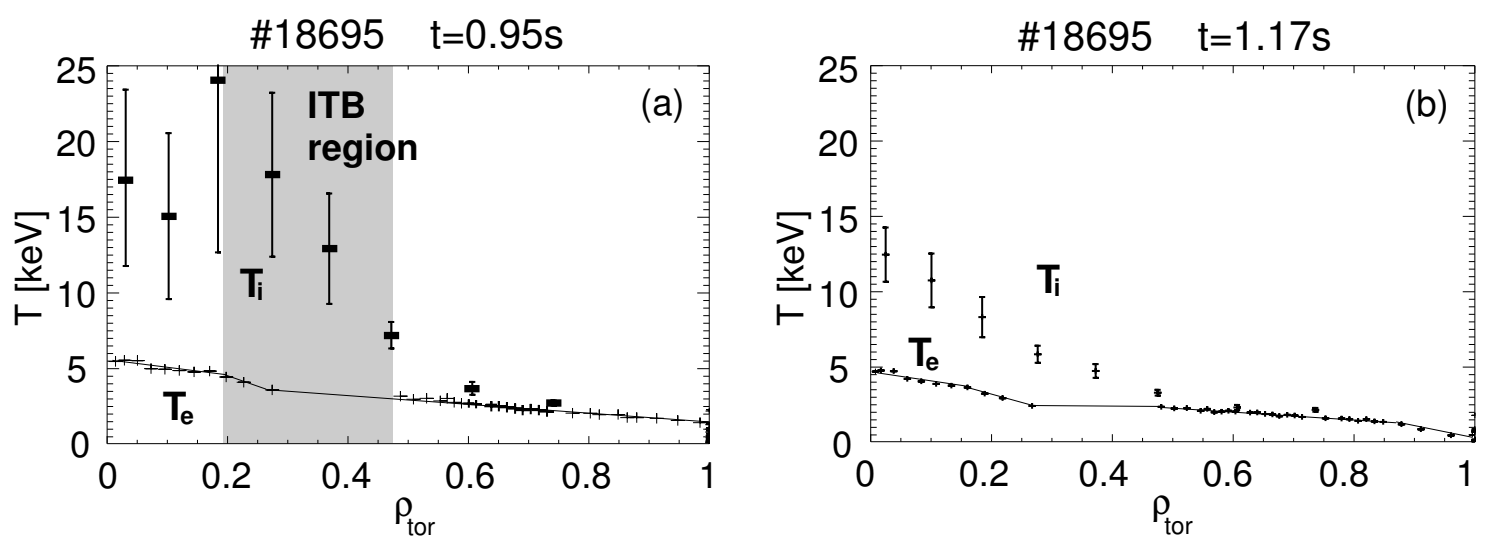

Figure 2. Temperature profiles of discharge \# 18695. (a) At 0.95 s, during the ITB phase. The shaded region has a high $\nabla T_{i}$. (b) At $1.17 \mathrm{~s}$, after the ITB collapse. Lines are a guide for the eye for $T_{e}$ profiles.

toroidal velocity is usually measured to be significantly higher during the ITB phase, as shown in Fig. 3 . The considerable improvement in core ion energy confinement is illustrated in Fig. 4. The good performance is also confirmed by $\beta_{N}$ values between 2 and 2.9 , H98(y,2) up to 1.9 and a moderate bootstrap current, around 35 $\%$ of the total plasma current. To detect events possibly associated with the ITB loss, it is useful to look at 

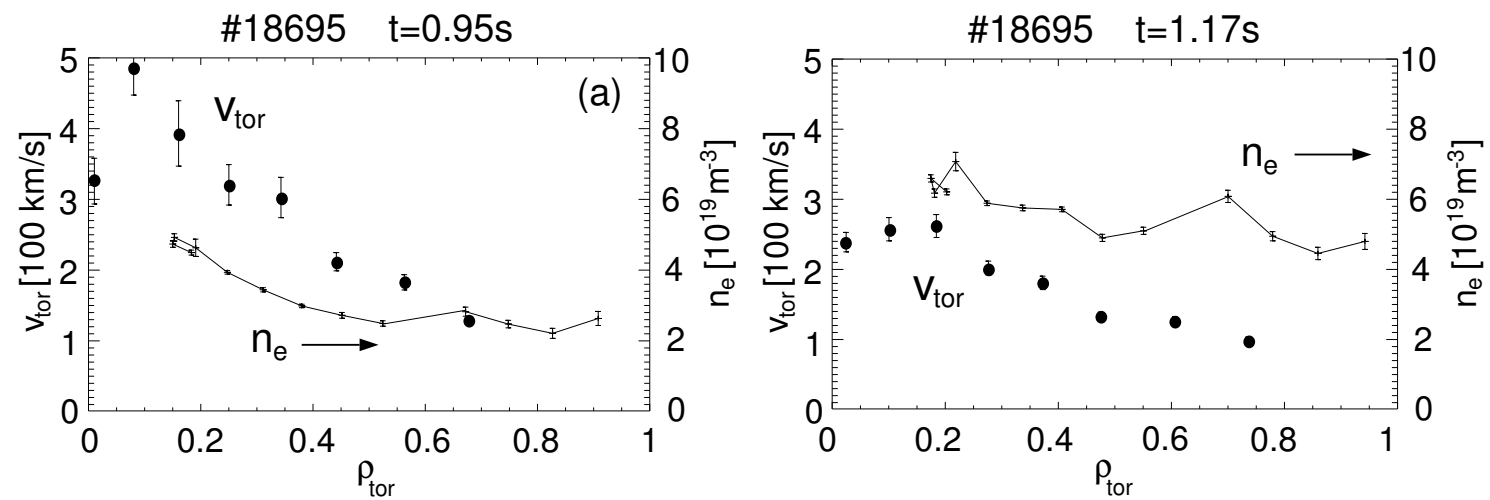

Figure 3. Density and toroidal velocity profiles of discharge \# 18695. (a) At 0.95 s, during the ITB phase. (b) At 1.17 s, after the ITB collapse.

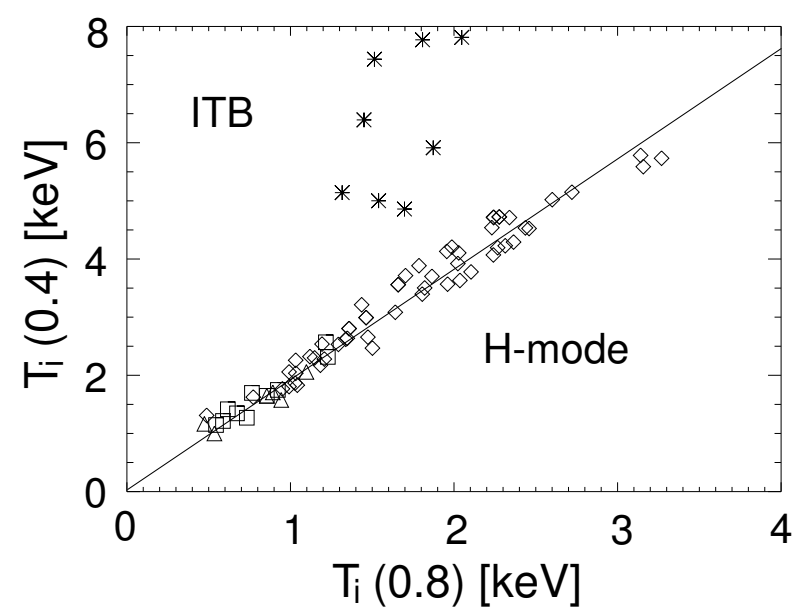

Figure 4. Core versus pedestal temperatures for ITB discharges (stars) compared to H-mode discharges (open symbols) signals with time resolution much faster than $20 \mathrm{~ms}$, the time resolution of the $T_{i}$ measurement. Correlations between fast signals help identifying the start of the ITB loss, marked with a dashed line in Fig. 1. Indeed, between a high and a low $T_{i}$ time values we always observe that the plasma energy stops increasing, although $n_{e}$ fuelling by the beams is still effective. At this time point, also a $T_{e}$ plateau is obeserved, correlated with a smooth increase of the D- $\alpha$ signal. The neutron rate starts decreasing as well.

\section{MHD activity and ITB}

Type I ELMs are believed to be a major candidate for the loss of the good energy confinement as it has been observed in JET [8]. Fast CXRS measurements allow to resolve the ITB decay with respect to the ELMs onset. In almost all ASDEX Upgrade cases ITBs are observed to collapse already before the first type I ELM occurs, 


\section{G. Tardini et al.}

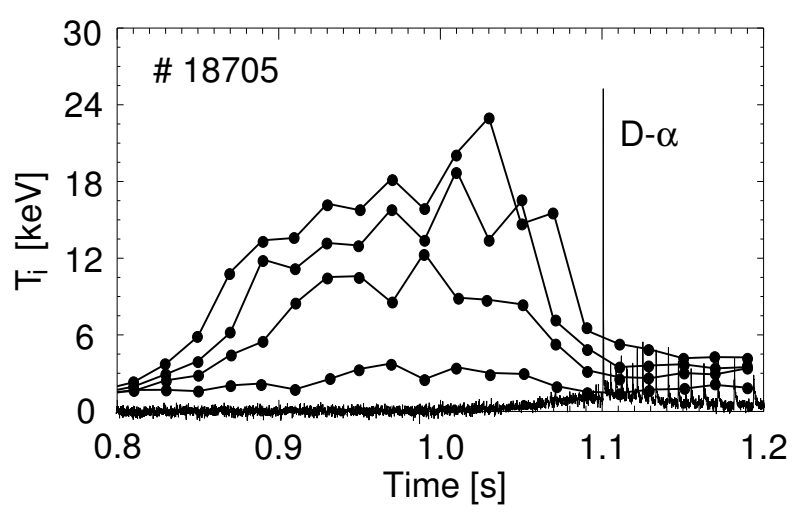

Figure 5. Timing of the ITB with respect to the ELM onset. The ITB phase is terminated clearly before the first ELM occurs

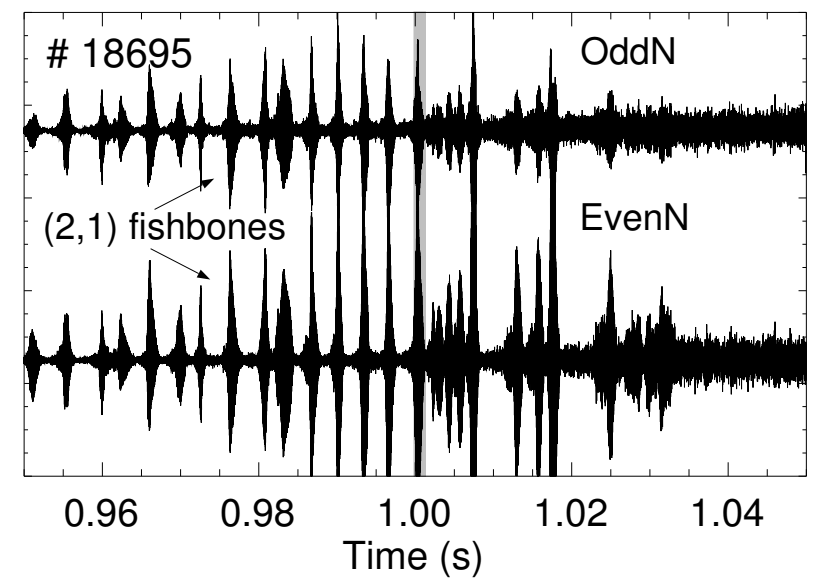

Figure 6. MHD activity during the ITB phase: fishbones and NTMs are observed. The activity varies strongly in time due to the rapid increase of the background density and the suprathermal ion population. The shaded vertical region indicates the beginning of the ITB collapse.

as shown in Fig. 5. Therefore, in general ELMs are not the cause of the confinement deterioration, although it is not ruled out that in some cases they might erode the transport barrier. The core MHD activity of the ITB discharges differs from discharge to discharge. Also within single experiments it varies strongly in time due to the rapidly increasing background $n_{e}$. In several discharges the current ramp-up is performed in two steps. Interestingly, they feature the fishbones instability (see Figure 6), which is at the same time an evidence for a significant amount of fast ions as well as a mechanism expelling fast ions from the plasma. Such discharges exhibit the longest-lived ITBs in the present database. Strong MHD activity, usually $(2,1)$ or $(3,1)$, is found in almost all discharges, limiting the core energy confinement. In particular, the highest amplitude occurs before 


\section{Article: Thermal ions dilution and ITG suppression in ASDEX Upgrade ion ITBs}

the ITB collapses. The confinement loss due to the MHD activity can contribute to the ITB loss. On the other hand, a confinement loss - especially if suprathermal particles are lost - reduces the free energy available to sustain the MHD mode.

Recent ASDEX Upgrade measurements of fast ion losses with the Fast Ion Loss Detector [9] document a correlation between the O-point of a Neoclassical Tearing Mode (NTM) and a sudden expulsion of fast ions from the plasma. Also fishbones, observed in some ITB discharges, are predicted to expell fast ions. The sudden drop in the neutron yield (Figure 1 (b)) is consistent with a loss of fast ions, although the $T_{i}$ profile variation affects significantly the neutron production as well. The drop in the neutron rate is well correlated in time with the increased heat load on the divertor indicated by the D- $\alpha$ signal (see Figure 1 (a)). Also the plateau behaviour of $T_{e}$, or even sudden drop in some discharges, is compatible with a fast ion loss, since electrons are heated earlier than ions, already at the beginning of the beam ions slowing down process. The importance of the fast ion population with respect to the ITB maintenance will be discussed in detail in the following sections.

\section{Fast ions and ion ITBs in the fluid picture}

In ASDEX Upgrade the criteria for the onset of internal transport barrier have been investigated statistically [6]. The ITG mode is commonly believed to be stabilised by high plasma rotational shearing rate, $\omega_{E \times B}$, by weak or negative magnetic shear, by the Shafranov shift and a high ratio $T_{i} / T_{e}$. In fact, the key parameter for ITB formation in AUG is found to be the low plasma density [6]. The role of $\omega_{E \times B}$ is also discussed in [6], although more specifically for ITBs relying on NBI pre-heating. It turns out that $\omega_{E \times B}$ is not enough to explain the ITB sustainment alone. In absence of NBI pre-heating its contribution should be marginal also at the ITB onset, because the plasma toroidal velocity builds up on a $\tau_{s d}$ time scale after the NBI is switched on. The toroidal velocity increases further, eventually, during the ITB phase. The magnetic shear is expected to be positive, because we apply neither off-axis current drive nor pre-heating in our discharges. The Motional Stark Effect measurement in Fig. 7 confirms the monotonic shape of the q profile within the experimental uncertainty, in particular at $\rho_{t} \approx 0.3-0.4$, the typical radial location of ASDEX Upgrade ion ITBs. A high ratio $T_{i} / T_{e}$ can also possibly stabilise ITG turbulence, but it is still below 2 immediately after the NBI switch on. Its role is, however, important in the ITB sustainment and will be discussed in detail in section $5 .$. 


\section{G. Tardini et al.}

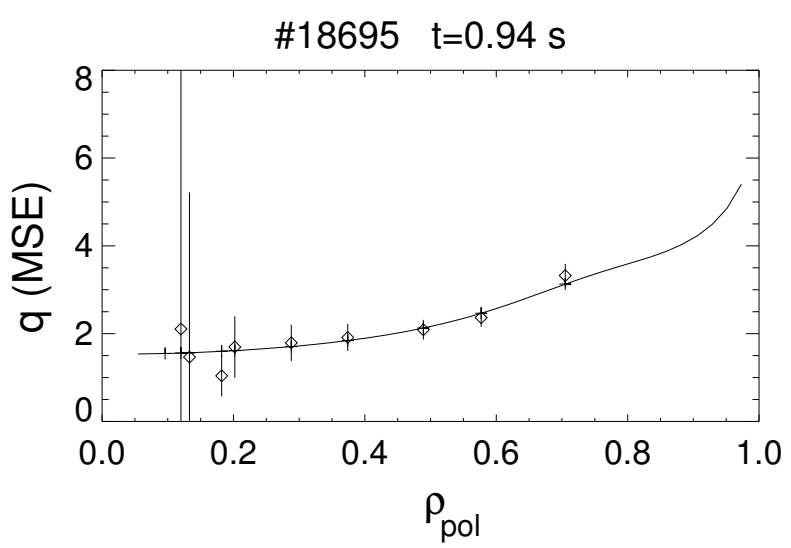

Figure 7. Safety factor profile from Motional Stark Effect measurements during the ITB phase. No reverse shear is observed in the ITB region. No off-axis current drive sources are used in these discharges.

Theory-based 1D fluid models are selected for time-dependent simulation of the ITB discharges: the Weiland [10], in the electrostaic version with 8 equations and collisionality included, and the retuned GLF23 [11] [12] models. Both are based on ITG and Trapped Electron Mode physics. A dilution approximation is assumed for impurities and for fast ions, such that only the thermal deuterium density $n_{D}=n_{e}-Z_{C} n_{C}-n_{f a s t}$ enters the computation of the transport coefficients. In fact, fast ions do not participate to the ITG dynamics of thermal ions due to their much faster magnetic drift frequency.

The fluid models are plugged into the ASTRA code [14], which uses a time independent pencil code for NBI modelling. The density profile and the ion and electron temperature are predicted self-consistently. The boundary condition of the calculation are the experimental temperature and density values at $\rho_{\text {tor }}=0.8$. Transport reduction due to $\omega_{E \times B}$ is imposed according to the respective standard implementations, reference [15] for the Weiland model and [12] with quench factor $\alpha_{e}=1$ for the GLF23 model. $\omega_{E \times B}$ was determined using the experimental toroidal velocity, the neoclassical poloidal velocity profile and the diamagnetic contribution from the self-consistent pressure profile. Current diffusion is computed self-consistently with the ASTRA 2D equilibrium solver, but MHD activity is not modelled. Fig. 8 shows that both models predict with accuracy the transport barrier with respect to its location, width, central temperature and duration. However, the models predict an immediate ITB formation, with a full development already within $30 \mathrm{~ms}$ after the beams are switched on (Fig. 8 a), whereas the experimental ITB is clearly visible only about $70 \mathrm{~ms}$ after the NBI onset. The electron temperature is slightly overestimated by the models in the transient phase, following the too early onset of the 
Article: Thermal ions dilution and ITG suppression in ASDEX Upgrade ion ITBs
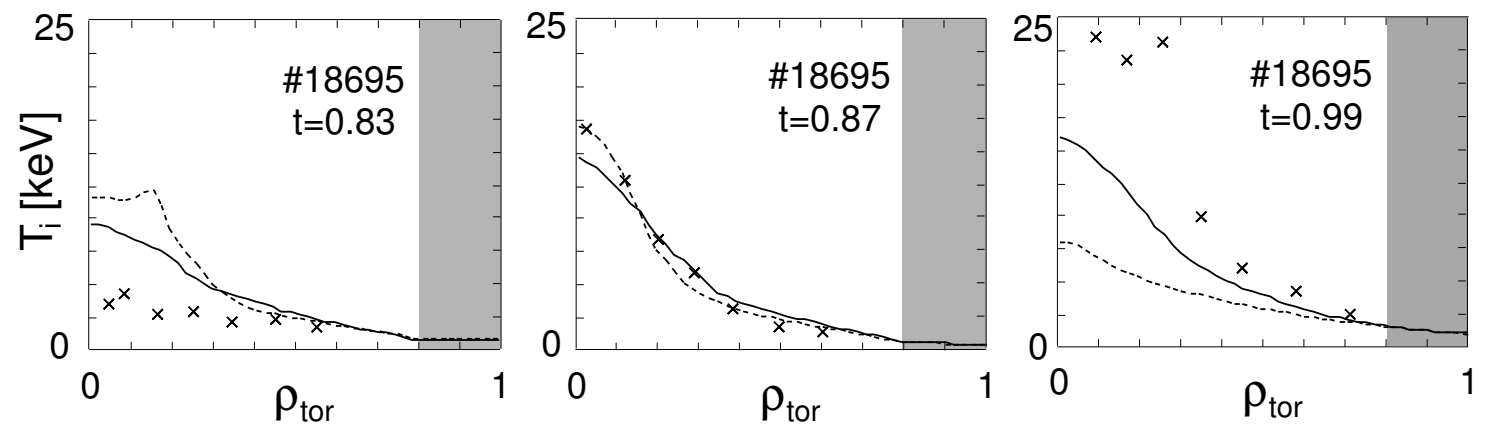

Figure 8. ASTRA $T_{i}$ simulations of discharge \# 18695 at different time points after the NBI is switched on at $0.8 \mathrm{~s}$. The solid line refers to the GLF23 mode, the dashed line to the Weiland model. The simulations neglect finite $\tau_{s d}$ of beam ions.
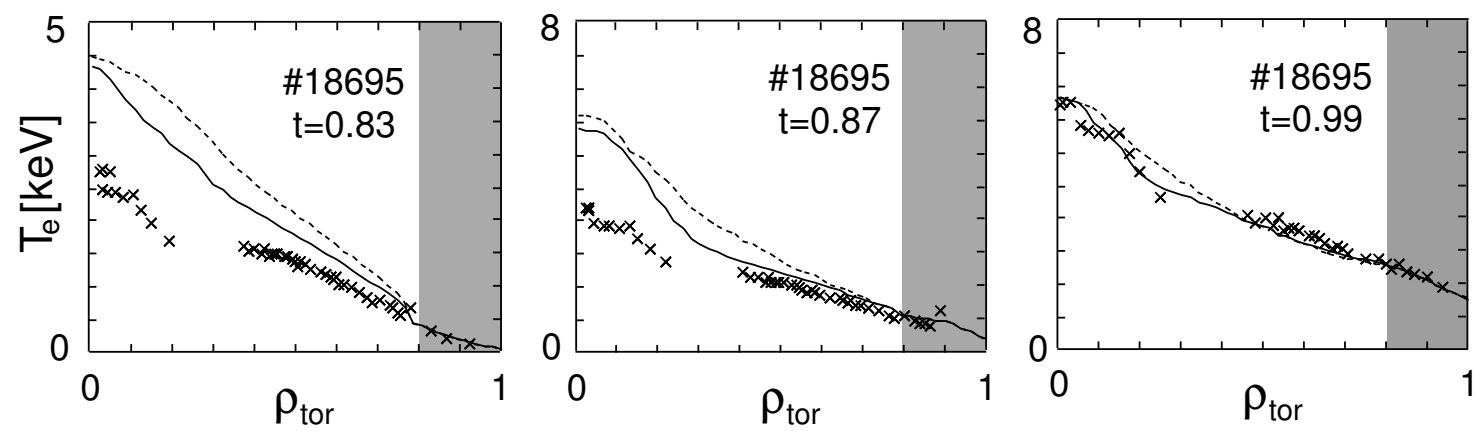

Figure 9. ASTRA $T_{e}$ simulations of discharge \# 18695. Time points and line code as in Fig. 8.

ion ITB. However, in no case an electron ITB is predicted, in agreement with the experimental observations. The plasma density, not shown here, is predicted accurately by both models. The shortcoming of the prediction of the ITB onset time is found to be due to the neglection of finite $\tau_{s d}$ in the ASTRA NBI package, $\tau_{s d}$ being about $100 \mathrm{~ms}$ in these experiments. As a consequence, the full NBI heating power is available in the simulations as soon as the beams are injected, whereas in the experiment it takes $\sim \tau_{s d}$ for the beam ions to transfer energy to the thermal plasma. This explains the overpredicted $T_{e}$ profiles. But more importantly, the ITG mode is suppressed in presence of a high value of the local fast ions fraction $n_{f a s t} / n_{D}$, which grows on a $\tau_{s d}$ time scale. This is found to be the key physics ingredient of the ITB formation in the simulations. This mechanism has a simple qualitative explanation in terms of ITG physics. Indeed, if $n_{\text {fast }}$ is a significant fraction of the background plasma density, thermal deuterons are too diluted to develop the ITG instability loop. As mentioned above, fast ions as well as impurities have different drift frequencies than deuterons and do not contribute to the ITG dynamics. On the contrary, the electrons stripped from beam neutrals (or impurities) are available to counteract 


\section{G. Tardini et al.}

the ion density perturbation which drives the feedback loop of the ITG instability [13]. The suprathermal ion population is fuelled as soon as the beams are injected and increases almost linearly in time during the first $\tau_{s d}$, leading to fast ion accumulation. Eventually, the thermalisation rate of beam fast ions compensates the injection rate, so that the total amount of fast ions is roughly constant (or even slowly decreasing) after $\tau_{s d}$ from the NBI onset. Therefore, also the ITG suppression becomes effective on a $\tau_{s d}$ time scale after the NBI onset. Within this dilution picture, a $n_{e}$ threshold for ITB formation at a given NBI power is predicted, in agreement with ASDEX Upgrade results [6]. In fact, high density reduces the ratio $n_{f a s t} / n_{e}$ at a given NBI power, it limits the beam penetration and shortens $\tau_{s d}$ and hence the amount of fast ion. This qualitative picture is reproduced by the fluid models, with a reasonable quantitative agreement with the experiments. In the next section, the more general gyrokinetic theory is applied to predict the critical value of the fast ion fraction to suppress the ITG instability. The GS2 code [16] is particularly suited to study the dilution effect quantitatively, because it takes into account the effect of the suprathermal ions on the ITG mode growth. In fact, whereas the fluid models use a simplified dilution assumption, so that fast ions are entirely neglected, GS2 allows to retain the fast ion population as a separate plasma species.

\section{Gyrokinetic calculations of the ITG linear stability}

A quantitative study of the of the fast ions fraction $n_{\text {fast }} / n_{e}$ required to ensure linear stability of the ITG mode is performed with the linear gyrokinetic code GS2 [16]. The runs are electrostatic and assume $\mathrm{k} \rho_{i}=$ 0.3, which corresponds to the typical ITG wavelength. Collisionality was not included, because the considered discharges exhibit very low density and electron temperature of several keV. The MHD $\alpha$ is taken from the ASTRA equilibrium, retaining the contribution of the fast ions pressure. The plasma parameters are taken from a typical ITB discharge at the barrier location, $\mathrm{r} / \mathrm{a}=0.3$, at the time when the barrier is about to form. $T_{e}$ is assumed to be $3 \mathrm{keV}, T_{i} 6 \mathrm{keV}$. The fast ion population is not, in reality, in thermal equilibrium but here it is treated as a separate plasma species with a temperature of $60 \mathrm{keV}$. This is a reasonable assumption considering the neutral beam energy, which is $93 \mathrm{keV}$ for three NBI sources and $60 \mathrm{keV}$ for one, and taking into account partial beem slowing down. In any case, a sensitive study has shown a weak dependence of the ITG growth rate on the ratio $T_{\text {fast }} / T_{e}$ in the range $T_{\text {fast }} / T_{e}>10$. The ITG growth rate decreases progressively 

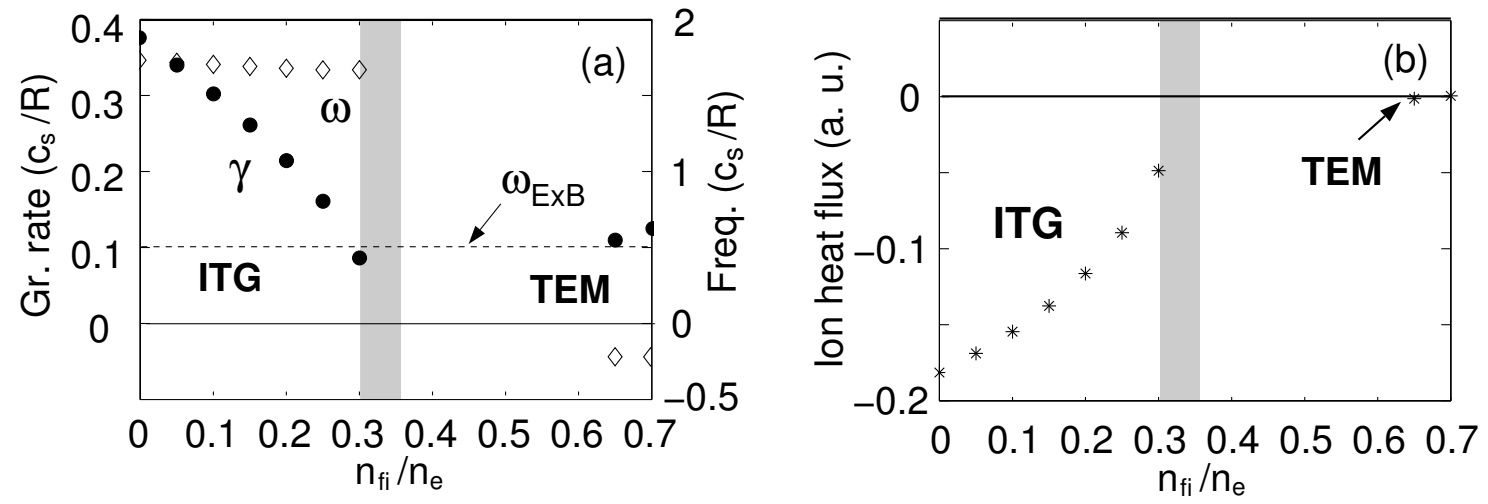

Figure 10. GS2 scan of $n_{\text {fast }} / n_{e}$ at ITB onset time: $\mathrm{k} \rho_{i}=0.3, \mathrm{r} / \mathrm{a}=0.3, \hat{s}=0.5, \alpha=0.01, T_{i} / T_{e}=2, T_{\text {fast }} / T_{e}=20$, $q=2, R / L_{n_{e}}=R / L_{n_{i}}=4, R / L_{T_{e}}=6, R / L_{T_{i}}=10, R / L_{T_{\text {fast }}}=0.5$, zero collisionality. (a) ITG growth rate: the mode is stable for $n_{\text {fast }} / n_{e}$ above $30 \%$. (b) Ion heat flux. Negative means outward flux. No ion heat is transported when the ITG mode is stabilised. The shaded region marks the contribution of the $\omega_{E \times B}$ stabilisation.

with increasing $n_{\text {fast }} / n_{e}$ until it is completely stabilised above a fraction of 30/35\% (Fig. 10 (a)), assuming the stability criterium $\omega_{E \times B}>\gamma_{I T G}[15]$. Fig. 10 (b) shows the ion heat flux decrease and suppression when the ITG mode is stabilised by increasing $n_{\text {fast }} / n_{e}$. The shaded regions in Fig. 10 indicates the shift of the critical ion fast fraction assuming the stabilisation criterium as in [15] and a value of $\omega_{E \times B}=0.1$ in units $c_{s} / R$, where $R$ is the tokamak major radius and $c_{s}=\left(T_{e} / m_{H}\right), m_{H}$ being the hydrogen mass. The assumption $\omega_{E \times B}=$ 0.1 is based on the typical value found taking $\omega_{E \times B}$ as described in paragraph 4 ., but it is of course a possible error source when determining the effective ITG stabilisation, due to the experimental uncertanties. Further significant uncertainties arise from $T_{i} / T_{e}$ and $R / L_{T_{i}}$, where $L_{f}$ for any radial profile $f$ is defined as $|f / \nabla f|$. In fact, $T_{i} / T_{e}$ and $R / L_{T_{i}}$ are determined within the experimental uncertainties and have a strong effect on the ITG stabilisation. Minor errors are due to the parameters $\hat{s}$, the Shafranov shift and $R / L_{n_{e}}$ (destabilising).

Once the transport barrier has formed, there is a trade off between stabilising and destabilising contributions. Indeed, higher $\nabla T_{i}$ destabilises the ITG mode, but $\omega_{E \times B}$ is higher as well as the Shafranov shift, due to stronger plasma rotation and plasma pressure, respectively. The most effective stabilising mechanism is provided by the ratio $T_{i} / T_{e}$, which can reach as high values as 5 during the ITB phase. Its impact on ITG stability is illustrated in Fig. 11. Full circles refer to $T_{i} / T_{e}=2$, empty symbols to $T_{i} / T_{e}=2.5$, all other parameters are kept unchanged. The critical value of $n_{\text {fast }} / n_{e}$ is reduced from $60 \%$ to $30 \%$. 


\section{G. Tardini et al.}

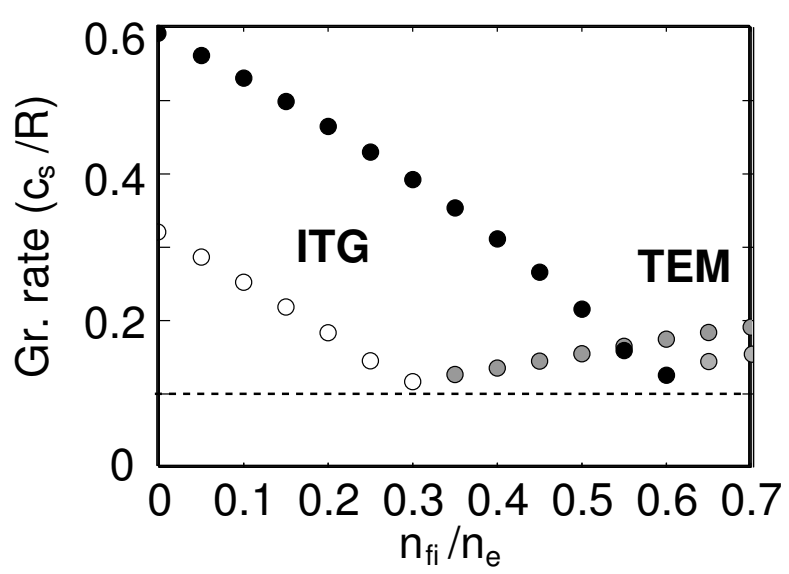

Figure 11. GS2 computation of the ITG growth rate with plasma parameters from the ITB phase: $R / L_{T_{i}}=15$, $R / L_{T_{e}}=7$, other parameters like in Figure 10. Changing the ratio $T_{i} / T_{e}$ from 2 (empty circles) to 2.5 (full circles) has a strong stabilising impact on the ITG mode.

\section{NBI Monte Carlo modelling}

An accurate time-dependent NBI reconstruction is required for a quantitative assesment on the local fast ion population in the plasma. This discriminates whether the ITG suppression mechanism described in section 4. is relevant under the experimental conditions. For this purpose, the TRANSP code has been implemented, in particular the NBI module NUBEAM [17]. The other NBI codes available at ASDEX Upgrade assume steady state and have been used for benchmark. Whereas GS2 calculations in this work are performed neglecting impurities in order to reduce the computing time, TRANSP runs retain carbon impurities, since $\mathrm{Z}_{e f f}$ affects the prediction of the beam slowing down process. Since an accurate measurement of the effective ion charge is not available, we assume conservatively a low estimate of $\mathrm{Z}_{e f f}=1.8$, constant in time and space. This means that $n_{C} \propto n_{e}$, with $n_{C} / n_{e} \approx 9 \%$. Other impurities have been neglected. The assumptions for the concentration of the impurity species add some uncertainty in the comparison with the GS2 preditcions. The active parameter which gives rise to the ITG instability growth is $\tilde{n}_{D, t h} / n_{e}$ where $\tilde{n}_{D, t h}$ is the perturbation of the thermal deuterium density. Thus, the relevant quantity to compare with the gyrokinetic calculations is the effective dilution, resulting by summing up the contributions from fast ions and impurities:

$1-\frac{n_{D, t h}}{n_{e}}=\frac{n_{\text {fast }}+\sum_{i} Z_{i m p, i} n_{i m p, i}}{n_{e}} \approx \frac{n_{\text {fast }}}{n_{e}}+\frac{n_{C}}{n_{e}} \approx \frac{n_{\text {fast }}}{n_{e}}+0.09$ 
Article: Thermal ions dilution and ITG suppression in ASDEX Upgrade ion ITBs

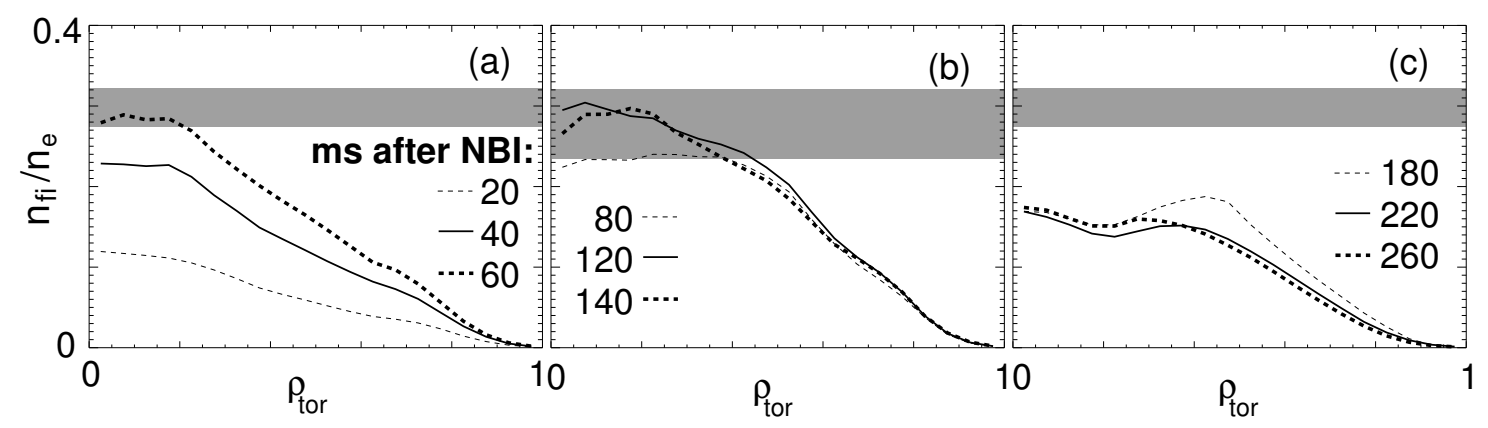

Figure 12. TRANSP time evolution of the fast ion concentration profiles for the discharge \#18695 after the NBI is switched on: (a) before the ITB, (b) during the ITB phase, (c) after the ITB. The shaded region indicates the gyrokinetic critical fast ion fraction for ITG suppression, with experimental uncertainty arising mainly from $\omega_{E \times B}$. A value of $9 \%$ due to carbon impurities has been deducted compared to Fig. 10.

The TRANSP runs have been performed taking the plasma parameters and background profiles from the experiment: $T_{i}$ and $v_{t o r}$ from the fast CXRS system, $T_{e}$ and $n_{e}$ from the core Thomson scattering. The equilibrium is computed by the internal solver, the boundary condition being the experimental equilibrium at $\rho_{v o l}=0.99$. Figure 12 shows the time evolution of the fast ion population normalised to the local electron density. The fast ions accumulate in the plasma as they get thermalised on a $\tau_{s d}$ time scale, which is about $80 \mathrm{~ms}$ in this discharge at NBI onset. Then their amount is roughly constant (slowly decreasing) in time, since the beam thermalisation and ionisation rates are balanced. However, beam thermalisation causes the background $n_{e}$ to grow, so that the fast ions fraction decreases in time. Additionally, growing $n_{e}$ reduces the beam penetration and shortens the slowing down time, thus reducing further the ratio $n_{f a s t} / n_{e}$. This behaviour is quantitatively described in Fig. 12 b) and c). The shaded region in Fig. 12 corresponds to the ITG threshold predicted by the GS2 calculation, shifted by the $9 \%$ contribution to dilution due to carbon impurities. The fast ions concentration predicted by TRANSP is perfectly consistent with the critical fraction for the ITB formation derived in section 5.. The critical values are reached locally up to $\rho_{t o r} \approx 0.35$, which matches the experimental ITB width with high accuracy. It is wortwhile noting that using the time dependent source terms and fast ion density profiles from the TRANSP calculations as input for the fluid models, no ITB formation is observed in the few test cases, including discharge \# 18695. The fast ion fraction required by the models to suppress the ITG is higher than the one obtained with TRANSP, possibly due to the simplified dilution assumption.

The timing of the ITB onset (Fig. 12 a)) is in good agreement with the experimental one. After a couple of 


\section{G. Tardini et al.}

slowing down times, the fast ions fraction is appearently too low to sustain the ITB, as shown in Fig. $12 \mathrm{c}$ ). This suggests a possible mechanism for the barrier collapse. A decrease of the fast ion fraction enhances ion heat transport. As a consequence, the ratio $T_{i} / T_{e}$ decreases, thus leading to further confinement loss. At the same time, $\omega_{E \times B}$ and the Shafranov shift drop as well as transport grows. This can develop a runaway mechanism which destroys the favourable confinement region. The confinement deterioration is stopped as soon as $\nabla T_{i}$ is not anymore strong enough to drive the ITG mode further unstable. The time scale of this process, estimated to be of the order of the confinement time in the ITB region, is consistent with the observed duration of the ITB collapse of 20-40 ms (Figures 1 and 5).

\section{ITG and dilution in tokamak plasmas}

While NBI is necessary to input the required amount of fast ions, thermalisation of beam ions on a $\tau_{s d}$ time scale causes an increase of the background density, possibly helped by a L to $\mathrm{H}$ transition. As a consequence, the ITB lifetime is intrinsicellay limited in time, unless a different scheme such as strong negative magnetic shear is applied. However, the current drive capabilities of ASDEX Upgrade allow a reversed q profile only close to the magnetic axis, via Electron Cyclotron Current Drive, which is localised but cannot drive high amounts of current. On the other hand, Neutral Beam Current Drive can drive a significant current, but not localised, so that the shape of the q-profile is hardly affected [18]. Replacing NBI power with ICRH would prevent the beam fuelling and the subsequent background density increase, but any attempts to produce ICRF heated ITBs in ASDEX Upgrade have failed so far. This is perfectly consistent with the dilution mechanism. In fact, ICRH produces highly energetic ions, such that for a given input power their amount is negligible compared to the background density. Their poor confinement in a medium size device like ASDEX Upgrade corrects the estimate of the fast ion fraction unfavourably. A further drawback of the dilution scheme is that even at the lowest achievable plasma densities the required fast ions fraction is never reached outside $\rho_{\text {tor }} \approx 0.35$. The narrow ITB width and the absence of a density ITB limit the bootstrap current fraction to moderate values of 30-40\%, making this scheme unsuited for non-inductive tokamak operation.

It is worthwhile noting that fast ions play a role not only when their amount is high enough to completely suppress turbulence. In fact, they always reduce the ITG growth rate almost linearly for $n_{\text {fast }} / n_{e}$ between 0 


\section{Article: Thermal ions dilution and ITG suppression in ASDEX Upgrade ion ITBs}

and the ITG suppression threshold, as shown in Fig. 10. Therefore, a quantitative evaluation of the benificial effect of thermal ions dilution is worthwhile on several advanced scenarios and tokamak devices. In particular it is interesting to quantify the confinement improvement for ASDEX Upgrade improved H-modes, which obtain better confinement at low plasma density and high NBI power. Only non-linear calculations can provide a reliable prediction, since one has to consider the ITG mode at saturation. The impact of the local fast ion concentration has been already invoked to explain the good confinement of TFTR supershots [19]. In particular, the authors emphasise the correlation between the energy confinement and the peaking of the neureal-beam fuelling profile. Interestingly, the correlation is shown to be much stronger for the ion stored energy than for electrons' [19]. A similar result is found on ASDEX Upgrade ITBs with 7.5 MW NBI input power [20]: if three on-axis sources (2.5 MW each) are applied at low plasma density, an ITB forms. If one central beam is replaced by an off-axis source, then the barrier does not occur. For $P_{N B I} \geq 10 \mathrm{MW}$ instead there is always enough central deposition to obtain an ITB. The ion dilution mechanism is mentioned explicitly to explain the Radiatively Improved (RI) discharges of the TEXTOR tokamak [21]. In that case $n_{D, t h} / n_{e}$ is reduced by the strong impurity content rather than by beam ions. The dilution of deuterons is shown to mitigate ion heat transport by reducing the ITG growth rate, but the mode is not entirely suppressed and no ITB arises. In JET ion ITBs the dilution effect is not expected to play a dominant role, due to the device size. It could be nevertheless a favourable ingredient for ITB formation which has not been taken into account so far. Turbulence suppression due to thermal ion dilution is expected to be inefficient in ITER. In fact, high voltage negative ion sources are expected to yield good beam penetration. However, due to the high energy per injected ion, for a given NBI power much less fast particles are injected than in present days positive ions sources. Additionally, the large device size prevents the local accumulation of suprathermal ions. Fusion $\alpha$-particles contribute to thermal ions dilution too, but the fraction of $n_{\alpha} / n_{e}$ planned for ITER-FEAT ranges between 0.001 and 0.008 [2]. Although each $\alpha$ particle contributes with two electrons, the ITER-FEAT planned value is at least one order of magnitude too low for any significant dilution effect. Moreover, the expected ratio $T_{i} / T_{e} \approx 1$ enhances significantly the $n_{\text {fast }} / n_{e}$ threshold of ITG suppression, making the dilution scheme for ITB formation far from applicable. 


\section{G. Tardini et al.}

\section{Conclusions}

A new mechanism of ion ITB formation is identified and quantitatively investigated. The ITG mode is prevented by thermal ions dilution due to the high amount of fast ions in presence of strong NBI injection at low plasma density. This ITBs are easily reproducible [5], unlike older barriers relying on pre-heating and q-profile control. The scenario is shown to be robust and to be hardly affected by the q-profile: changing the timing of the NBI heating, the plasma current, the magnetic field and the current ramp up rate leads always to ITB formation, provided the plasma density is low $\left(2-310^{19} \mathrm{~m}^{-3}\right)$. Although the discharges differ for the MHD activity and $\mathrm{q}_{95}$, the ITBs exhibit to large extent the same behaviour.

The dilution mechanism explains the phenomenology of ASDEX Upgrade ion ITBs in absence of pre-heating. The ITB width is predicted to be up to $\rho_{t o r} \approx 0.35$, as observed experimentally. Also the measured ITB onset time is consistent with the build up time of the fast ions population, of the order of the slowing down time. The empirical result that high NBI power is required for ASDEX Upgrade ion ITBs finds a physics basis. The density threshold for ITB formation documented in $[6]$ is explained by the variation of the dilution factor $n_{f a s t} / n_{e}$. This parameter explains also the ITB lifetime, of the order of $\tau_{s d}$, as the fast ions get thermalised and enhance the background density. A runaway mechanism involving also $T_{i} / T_{e}, \omega_{E \times B}$ and the Shafranov shift is proposed. It is consistent with the duration of the ITB collapse process. According to the dilution mechanism, the confinement loss does not need to be triggered by a MHD event, but it can, due to fast ion losses and possibly also to confinement degradation. The dilution mechanism explains also why attempts of ICRH heated ITBs have failed on ASDEX Upgrade so far.

Gyrokinetic linear stability analysis with the GS2 code predicts a threshold in $n_{\text {fast }} / n_{e}$ for ITG suppression. The stabilising role of $T_{i} / T_{e}$ and $\omega_{E \times B}$ is shown to play an important role for ITB formation and sustainment. TRANSP simulations of the time dependent beam ions dynamics allow a quantitative assessment of the fast ion populations at different radial locations.

The advantage of this scheme is that no q-profile control is required. However, while NBI is necessary to input enough fast ions, the background density increase effectively limits the ITB duration and width. The dilution mechanism can be related to the experimental observations of TFTR supershots [19] and is explicitely invoked to explain the Radiative Improved mode of the TEXTOR tokamak [21]. Here for the first time the mechanism 


\section{Article: Thermal ions dilution and ITG suppression in ASDEX Upgrade ion ITBs}

is associated with ITB formation. The role of dilution by partial suppression of the ITG turbulence in several tokamak plasmas is still to be quantitatively analysed, such as hybrid scenarios at low density and high power, as well as ion ITBs at JET. For ITER the dilution mechanism is predicted to be widely insufficient for ITB formation, so presently no obvious method is known to develop an ITB scenario in ITER without strong and localised current drive capability.

\section{References}

[1] G. Tardini et al., Nuclear Fusion 42 (2002) 258

[2] ITER Physics Basis, Nuclear Fusion 39 (1999) 2137

[3] R. C. Wolf et al., Plasma Phys. Control. Fusion 45 (2003) R1-R91

[4] R. C. Wolf et al., Nuclear Fusion 41 (2001) 1259

[5] A. G. Peeters et al., in Fusion Energy 2000 (Proc. 18th Int. Conf. Sorrento, 2000) (Vienna: IAEA) CD-ROM EXP5/6 and http://www.iaea.org/programmes/ripc/physics/fec2000/html/node1.htm

[6] E. D. Quigley et al., Nuclear Fusion 44 (2004) 1189

[7] H. Zohm et al., 29th EPS Conference on Plasma Physics, 2002 Montreaux, P1.043

[8] JET Team (presented by A.C.C. Sips), Nuclear Fusion 41 (2001) 1559

[9] M. Garcia-Muñoz et al., 32th EPS Conference on Plasma Physics, 2005 Tarragona, P5.085

[10] J. Weiland et al, Nuclear Fusion 29 (1989) 1810

[11] R. E. Waltz et al, Physics of Plasmas 4 (1997) 2482

[12] J. Kinsey et al, Physics of Plasmas 12 (2005) 062302

[13] J. Weiland, Collective modes in Inhomogeneous Plasmas - Kinetic and Advanced Fluid Theory, Institute of Physics Publishing, Bristol (2000)

[14] G. V. Pereverzev, P. N. Yushmanov, IPP report 5/98 (2002)

[15] T. S. Hahm, K. H. Burrell, Physics of Plasmas 2 (1995) 1648

[16] M. Kotschenreuther et al., Physics of Plasmas 2 (1995) 2381

[17] A. Pankin, D. McCune, R. Andre et al., Comp. Phys. Comm. 159, No. 3 (2004) 157

[18] S. Günter et al., 32th EPS Conference on Plasma Physics, 2005 Tarragona, P4.075

[19] H. K. Park et al., Nuclear Fusion 37 (1997) 629-642

[20] A. Stäbler et al., 29th EPS Conference on Plasma Physics, 2002 Montreaux, O5.03 
G. Tardini et al.

[21] M. Z. Tokar et al., Plasma Phys. Control. Fusion 41 (1999) L9-L15 